\title{
Role of pairing interaction in neutron rich odd and even $\mathrm{Zr}$ nuclei
}

\author{
Madhubrata Bhattacharya and G. Gangopadhyay* \\ Department of Physics, University of Calcutta \\ 92, Acharya Prafulla Chandra Road, \\ Kolkata-700 009, India
}

\begin{abstract}
Neutron rich Zr nuclei with number of neutrons between $N=50$ and 82 are investigated in the relativistic mean field approach in co-ordinate space. The resonant levels in the positive energy continuum have been explicitly included in the calculation. Odd nuclei have been treated in the blocking approximation. Our calculation indicates that the dripline for odd mass isotopes is far away from that for the even mass ones. Pairing interaction plays a significant role in stabilizing the even isotopes, thus extending the dripline for them.

PACS numbers: 21.60.Jz, 27.60.+j
\end{abstract}

Exotic nuclei very close to the neutron drip line exhibit a number of new and interesting features such as neutron skin and halo, rearrangement of magic numbers, quenching of spin orbit splitting, etc. The last bound neutrons in such a nucleus lie very close to the continuum. Hence, the effect of the positive energy continuum on the structure of such nuclei needs to be studied carefully. Another very important aspect of nuclei near the drip line is the additional stability contributed by the pairing. This effect may be studied by investigating the odd mass nuclei along with the even mass ones. Although a number of such investigations has been undertaken in lighter mass regions, nuclei in the the medium and the heavy mass regions, have not yet been examined in sufficient detail.

In the present work, we study $\mathrm{Zr}$ isotopes using relativistic mean field (RMF) approach in co-ordinate space. Though the RMF equations are usually solved by expanding them in a harmonic oscillator basis, it is well known that this method cannot explain the density in halo nuclei near the drip line because of slow convergence. Relativistic Hartree Bogoliubov method in co-ordinate space (RCHB) has been found to be a very accurate method of treating the nuclei very close to the drip line. The effect of the states in the continuum has been incorporated in most of these calculations by solving the equations in co-ordinate space where the continuum has been replaced by a set of discrete positive energy states with the box normalization condition. However, the single particle energy levels depend on the size of the box chosen in this scenario, an unwelcome complication.

Nonrelativistic mean field equations involving continuum states have already been solved with exact boundary conditions 1, 2 for zero range and finite range pairing forces. Corresponding relativistic calculations have also been performed [3, 4] with exact boundary conditions. All these calculations have taken into account the width of the continuum levels also. We have also used the RMF calculation in co-ordinate space including the continuum states to study neutron rich $\mathrm{C}$ and $\mathrm{Be}[\underline{5}]$ and $\mathrm{Ca}$ and $\mathrm{Ni}$ nuclei $[\underline{6}]$. We find that this method gives results in very good agreement with the more complicated relativistic Hartree Bogoliubov (RHB) approach.

In the present work, the structure of even and odd Zr isotopes with neutron number between $N=50$ and 82 have been studied in the relativistic mean field formalism with exact boundary conditions, Numerous authors have studied neutron rich even mass $\mathrm{Zr}$ isotopes up to the neutron drip line using relativistic and nonrelativistic mean field approaches. We cite only a few works published recently. Sharma et al. 7] first applied RMF to study neutron rich Zr nuclei up to the drip line. RHB approach was also utilized to study some of the neutron rich isotopes [8, [9]. Zhang et al. [10] used RCHB approach to study Zr and some other proton closed shell nuclei. Deformed RMF approach was used by Geng et al. to study even-even Zr nuclei[11]. RCHB approach was applied to study ${ }^{134} \mathrm{Zr}[12]$. Pearson[13] compared the results of Skyrme HF and RMF calculations for even-even neutron rich Zr nuclei. Recently co-ordinate space Hartree-Fock-Bogoliubov calculations were performed by Blazkiewicz et al. 14 for even-even $\mathrm{Zr}$ isotopes. They found that the neutron drip line is at $N=82$. Continuum RMF calculation with exact boundary conditions has been used to study $\mathrm{Zr}$ isotopes [3]. Zhang et al. [15] have studied ${ }^{122} \mathrm{Zr}$ in the continuum relativistic mean field theory using the analytic continuation approach for single-particle resonance. The authors of the last two works have used the relativistic force NLSH[16] though Sandulescu et al. have concluded that the results are not much different even if one uses NL3[17] or TM1 18]. They have studied the even-even nuclei beyond $\mathrm{N}=82$. However, we have come across no mean field calculation in neutron rich odd mass Zr nuclei.

As already mentioned, the aim of the present work is to study the role of the pairing as well as the resonant states in neutron rich Zr nuclei, particularly with respect to odd mass nuclei near the neutron drip line. We have earlier seen [6] that in the case of Ca isotopes, the drip line for the even mass nuclei lies far beyond the same for odd mass ones. 
However, for $\mathrm{Ni}$ isotopes, the dripline nuclei for both even and odd masses are adjacent. This phenomenon has been interpreted as the effect of pairing correlations stabilizing the even-even nuclei in the case of levels with appreciable occupation factor lying very close to or even inside the positive energy continuum. This scenario is observed in the single particle energy levels in $\mathrm{Ca}$ but not in $\mathrm{Ni}$ isotopes. In the present work we extend our study to odd mass $\mathrm{Zr}$ nuclei.

Relativistic mean field theory is well known and will not be detailed here. In the present work, we have mainly employed the force NL3[17]. This force is known to provide good results for binding energy and radius throughout the periodic table. For comparison, we have also redone the calculations with the force NLSH[16]. Spherical symmetry has been assumed for all the nuclei as the protons form a closed shell. It is likely that some nuclei in the chain are deformed and also possible that the actual position of the neutron drip line may vary slightly on inclusion of deformation. However, our aim is to investigate a possible difference in the location of the drip line in even and odd mass nuclei. Our conclusions in this regard, we believe, are more robust and not likely to be affected by inclusion of deformation to any large extent.

A delta interaction has been used for the pairing correlations between neutrons, i.e. $V=V_{0} \delta\left(\vec{r}_{1}-\vec{r}_{2}\right)$. The usual BCS equations now contain contributions from the bound states as well as the resonant continuum. The equations involving both these types of states have already been obtained [19, 20] and have been referred to as resonant-BCS (rBCS) equations. We have included the effect of the width of the positive energy levels. These equations have been solved in the co-ordinate space on a grid of size $0.08 \mathrm{fm}$. The positive energy resonance solutions are obtained using the scattering approach. All the negative energy states beyond $\mathrm{N}=40$ as well as the positive energy states for which resonance solutions have been found have been included in the rBCS calculation. We have assumed that beyond 20 $\mathrm{fm}$, the effect of nuclear interaction vanishes. We choose $V_{0}=-575 \mathrm{MeV}$ for the strength of the delta-interaction as this gives very good results for binding energy of even even $\mathrm{Zr}$ nuclei close to the stability valley. Odd nuclei have been treated in the blocking approximation corresponding to different single particle states.

The ground state spin-parity of the odd isotopes are known up to $N=63$, i.e. ${ }^{103} \mathrm{Zr}$. In our calculation for odd isotopes, the ground state spin parity values come out to be $5 / 2^{+}$up to ${ }^{97} \mathrm{Zr}, 1 / 2^{+}$for nuclei between ${ }^{99} \mathrm{Zr}$ and ${ }^{111} \mathrm{Zr}$ and $11 / 2^{+}$beyond that. We find that our calculations can correctly explain the ground state spin-parity for nuclei up to ${ }^{95} \mathrm{Zr}$ and ${ }^{99} \mathrm{Zr}$. In ${ }^{97} \mathrm{Zr}$, the ground state has actual spin parity $1 / 2^{+}$. Our calculation predicts the ground state to be $5 / 2^{+}$though there is another state with spin parity $1 / 2^{+}$approximately $270 \mathrm{keV}$ above this state. Similarly, the ground state in ${ }^{101} \mathrm{Zr}$ is predicted to be $1 / 2^{+}$. The spin parity of the actual ground state is $3 / 2^{+}$. The corresponding theoretical state is at an excitation energy of $530 \mathrm{keV}$. Our model fails to explain the observed negative parity of the ground state of ${ }^{103} \mathrm{Zr}$ which may possibly be due to deformation. Finally, we should mention that there are some doubts to the experimental spin-parity assignment to the ground states of ${ }^{99,101,103} \mathrm{Zr}$.

To check whether our results are dependent on the force selected, all the calculations have been redone using the force NLSH. Even Zr nuclei have already been studied using this force in the present model by Sandulescu et al. [3]. Following them we have taken $V_{0}=-275 \mathrm{MeV}$. In all the odd isotopes, the results for the ground state spin parity remain unchanged. Particularly in ${ }^{97} \mathrm{Zr}$, our calculation gives the ground state to be $5 / 2^{+}$where it is about $290 \mathrm{keV}$ below the $s_{1 / 2}$ state.

The preceding results show that the the ground state spin is correctly reproduced in lighter nuclei. In Table \ we present some of our results for one-quasiparticle states in the lighter nuclei where some information is available in the low energy regime, i.e. ${ }^{91,93,95} \mathrm{Zr}$. The experimental values are for the one-quasiparticle levels built on the corresponding states given in table. For example, in ${ }^{93} \mathrm{Zr}$ a $3 / 2^{+}$state has been observed at $0.26 \mathrm{MeV}$ by $(\mathrm{d}, \mathrm{p})$ reaction. However, study [23] suggests that it is in reality a three-quasiparticle state with $\left(2 d_{3 / 2}\right)_{3 / 2^{+}}^{3}$ configuration and the one-quasiparticle state actually corresponds to the $3 / 2^{+}$level at $1.4 \mathrm{MeV}$. It is seen that the above results for ground state spin parity values as well as for the other low energy one-quasiparticle states do not vary substantially if the force is changed from NL3 to NLSH. Henceforth, all the results to be presented are for the force NL3 unless otherwise mentioned. We also observe that the experimental values are reproduced reasonably well in most cases. One has to remember that in odd nuclei, often there is some contribution from the three-quasiparticle configurations even to low lying states and thus the experimental results may not agree with mean field theory without taking this configuration mixing into account.

In Table II the calculated and experimental or estimated binding energy values for the odd and even Zr isotopes between $N=50$ and 82 are presented. For comparison, the results for even-even nuclei from RCHB approach of Zhang et al. [10] using the force NLSH are also given. One can see the excellent overall agreement between experiment and theory particularly for the even isotopes in our calculation. In Fig. 1, we plot the one and two neutron separation energy for the nuclei studied. One neutron separation energy for a nucleus with neutron number $N$ has been written as $S_{n}=$ B.E. $(Z, N)-$ B.E. $(Z, N-1)$, and similarly for two neutron separation energy $S_{2 n}$. It is true that some of the $S_{n}$ values are large compared to the experiment. Besides, recent high precision mass measurements [22] show interesting structures beyond $N=56$. However, nuclear structure calculations in this regard are often not exact and 
should remember that the effects of deformation and configuration mixing have not been included which may affect the results. The most interesting aspect of the above results is the dripline for odd isotopes.

It is well known that relativistic theories predict that the dripline for even $\mathrm{Zr}$ nuclei is around $N=96$. We have confirmed that our calculations agree with them. In the case of odd isotopes, we find that the dripline is at $N=77$ for the force NL3. It is true that the location of the drip line is not exactly fixed in view of the assumptions in calculation, viz. neglecting the deformation, disregarding the effect of three-quasiparticle configurations, etc. However, the general result on the position of the drip line are expected to remain unaltered. Also interesting is the fact that the force NLSH predicts the dripline for odd nuclei to be at $N=81$. This is due to the use of a pairing strength value only half as large as that of the NL3 calculation. As explained later, because of the larger pairing strength, the even mass nuclei are more bound in the latter case and consequently the dripline for odd nuclei is at a lower mass. Though the two results do not agree exactly, we see that out essential conclusion that the driplines for the even and odd mass isotopes are far from each other, remains unaltered. In our earlier study involving odd mass Ca and Ni isotopes, we found that the dripline in odd nucleus is sensitively dependent on the pairing interaction as well as the single particle levels in neutron rich nuclei. Any reordering in the single particle level structure may introduce a corresponding change in the magic number in that mass region. The pairing interaction also plays an important role stabilizing the even-even isotopes compared to the neighbouring odd mass ones.

We plot the correlation energy due to pairing obtained in our calculation in Fig. 2. The correlation energy $E_{p}$ has been defined as the difference between the binding energy values the cases where pairing interaction has been taken into account and where it has been neglected. In odd nuclei, the ground state spin-parity for the solution when the pairing has been switched off may not agree with that when pairing is present. For example, in ${ }^{111} \mathrm{Zr}$, the extreme single particle model suggests that the last neutron should be in the $1 h_{11 / 2}$ state with the $3 s_{1 / 2}$ being completely filled. However, calculations predict that this corresponds to actually an excited state. The ground state has a neutron pair in the $1 h_{11 / 2}$ state and the odd neutron in the $3 s_{1 / 2}$ state. The correlation energy for a pair of neutron in the high spin state is very large, thus pushing down the latter configuration to be the ground state. The odd-even staggering is clearly visible, particularly in the case of nuclei with $N \geq 113$, where the last odd neutron occupies the $1 h_{11 / 2}$ single particle orbit in the ground state. This large difference in the pairing energies in even and odd isotopes can explain the additional stability of the even-even isotopes. In nuclei with $N \geq 113$, the correlation energy for odd and even isotopes differ by more than $3 \mathrm{MeV}$. This is sufficient to bind the even-even isotopes though the odd isotopes beyond $N=117$ are unstable against one neutron emission.

One interesting observation emerging from correlation energy results is the reduction in the magic gap at $N=82$. The correlation energy does not vanish but has a value of $1.65 \mathrm{MeV}$. One also sees that the correlation energy decreases at $N=70$. In Fig. 3, we plot the single particle levels near the Fermi level for the even isotopes. One can see that the large gap observed at $N=82$ in nuclei near the stability valley is not present. Actually, in neutron rich nuclei, the spin orbit splitting is reduced and the $1 h_{11 / 2}$ level is higher in the $\mathrm{Zr}$ nuclei studied. So we observe smaller gaps at $N=70$ and $N=82$ rather than a single large gap at the conventionally known magic number $N=82$. Such a rearrangement of the single particle orbitals is also observed for the NLSH results.

One of the interesting predictions in neutron rich nuclei is this decrease in the spin-orbit splitting. Indeed it is particularly this effect that leads to the prediction and, in light nuclei, observation of emergence of new magic numbers along with disappearance of old ones. Though we have not studied the drip line for even even isotopes which, as we have observed, is far away form the isotopes we have studied, a decrease in the spin orbit splitting is already evident. In Fig. 4, we have plotted the splitting for these nuclei. Here we have defined $E_{l s}=\left|E_{l+1 / 2}-E_{l-1 / 2}\right| /(2 l+1)$. It is clear that for high spin orbitals, the splitting falls off sharply with increase in the number of neutrons.

Neutron rich Zr nuclei with number of neutrons between $N=50$ and 82 are investigated in the relativistic mean field calculation in co-ordinate space. The resonance levels in the positive energy continuum have been explicitly included in the calculation by using the scattering approach. Odd nuclei have been treated in the blocking approximation A zero range force has been used for interaction between neutrons. Our calculation indicates that the odd mass neutron dripline $\mathrm{Zr}$ nucleus is ${ }^{117} \mathrm{Zr}$. It is far away from the even mass dripline nucleus ${ }^{136} \mathrm{Zr}$ predicted by earlier studies. The pairing interaction plays a significant role in stabilizing the even isotopes. The spin orbit splittings for the high spin single particle orbitals decrease drastically for large neutron excess, thus leading to a reduction in the magic gap an $N=82$.

This work was carried out with financial assistance of the Board of Research in Nuclear Sciences, Department of Atomic Energy (Sanction No. 2005/37/7/BRNS), Government of India.

[1] M. Grasso, N. Sandulescu, N. Van Giai, and R.J. Liotta, Phys. Rev. C 64, 064321 (2001). 
[3] N. Sandulescu, L.S. Geng, H. Toki, and G. C. Hillhouse, Phys. Rev. C68, 054323 (2003).

[4] Li-gang Cao, and Zhong-yu Ma, Nucl. Th. arxiv 0307016 (2003).

[5] G. Gangopadhyay, and S. Roy, J. Phys. G 31, 1111 (2005).

[6] M. Bhattacharya, and G. Gangopadhyay, Phys. Rev. C 72, 044318 (2005).

[7] M.M. Sharma, G.A. Lalazissis, W. Hillebrandt, P. Ring, Phys. Rev. Lett. 72, 1431 (1994).

[8] J. Meng, and P. Ring, Phys. Rev. Lett. 80, 460 (1998).

[9] T. Gonzalez-Llarena, J.L. Egido, G.A. Lalazissis, and P. Ring, Phys. Lett. 379B, 13 (1996).

[10] S.Q. Zhang, J. Meng, H. Toki, I. Tanihata, and S.-G. Zhou, Science in China Series G46, 632 (2003).

[11] L. Geng, H. Toki, S. Sugimoto, J. Meng, Prog. Theor. Phys. (Kyoto) 110, 921 (2003).

[12] J. Meng, Nucl. Phys. A654, 702c (1999).

[13] J.M. Pearson, Phys. Lett. 513B, 319 (2001).

[14] A. Blazkiewicz, V.E. Oberacker, A.S. Umar, and M. Stoitsov, Phys. Rev. C 71, 054321 (2005).

[15] S.S. Zhang, J. Meng, S.G. Zhou, and G.C. Hillhouse, Phys. Rev. C 70, 034308 (2004).

[16] M.M. Sharma, M.A. Nagarajan, and P. Ring, Phys. Lett. B312, 377 (1993).

[17] G.A. Lalazissis, J. König, and P. Ring, Phys. Rev. C55, 540 (1997).

[18] Y. Sugahara, and H. Toki, Nucl. Phys. A579, 557 (1994).

[19] N. Sandulescu, R.J. Liotta, and R.Wyss, Phys. Lett. 394B, 6 (1997).

[20] N. Sandulescu, N. Van Giai, and R.J. Liotta, Phys. Rev. C 61, 061301 (R) (2000).

[21] G. Audi, A.H. Wapstra, and C. Thibault, Nucl. Phys. A729, 337 (2003).

[22] U. Hager, et al., Phys. Rev. Lett. 96, 042504 (2006).

[23] C.R. Bingham, and G.T. Fabian, Phys. Rev. C7, 4 (1973). 
TABLE I: Excitation energies (in $\mathrm{MeV}$ ) of one-quasiparticle states in ${ }^{91,93,95} \mathrm{Zr}$. State refers to the single particle state on which the one-quasiparticle state is built.

\begin{tabular}{rcccc}
\hline A & State & NL3 & NLSH & Expt. \\
\hline $2 d_{3 / 2}$ & 1.9 & 1.9 & 2.0 \\
91 & $3 s_{1 / 2}$ & 1.7 & 1.6 & 1.2 \\
& $1 g_{7 / 2}$ & 1.1 & 1.1 & 1.8 \\
\hline $2 d_{3 / 2}$ & 1.5 & 1.5 & 1.4 \\
93 & $3 s_{1 / 2}$ & 1.2 & 1.2 & 0.9 \\
& $1 g_{7 / 2}$ & 1.1 & 1.0 & 1.4 \\
\hline & $2 d_{3 / 2}$ & 1.1 & 1.2 & 1.1 \\
95 & $3 s_{1 / 2}$ & 0.6 & 0.8 & 0.9 \\
& $1 g_{7 / 2}$ & 0.9 & 1.0 & 1.6 \\
\hline
\end{tabular}

TABLE II: Binding energy values in $\mathrm{Zr}$ isotopes. The values are in MeV. Here Expt. refers to either experimental or estimated values and are from [21] except for the nuclei with $\mathrm{A}=98-105$ where they are from [22]. The RMF column indicates the theoretical results obtained in the present work while RCHB refers to the Relativistic continuum Hartree Bogoliubov results of Zhang et al. [10].

\begin{tabular}{|c|c|c|c|c|c|c|c|}
\hline $\mathrm{A}$ & Expt. & $\overline{\mathrm{RMF}}$ & $\mathrm{RCHB}$ & $\mathrm{A}$ & Expt. & $\overline{\mathrm{RMF}}$ & $\overline{\mathrm{RCHB}}$ \\
\hline 90 & $\overline{783.892}$ & 783.432 & 783.173 & 107 & 887.565 & 889.238 & \\
\hline 91 & 791.806 & 789.145 & & 108 & 892.620 & 895.580 & 883.105 \\
\hline 92 & 799.721 & 799.802 & 795.603 & 109 & 895.672 & 898.198 & \\
\hline 93 & 806.456 & 804.940 & & 110 & 900.460 & 904.816 & 891.983 \\
\hline 94 & 814.677 & 814.566 & 807.665 & 111 & & 906.286 & \\
\hline 95 & 821.139 & 819.248 & & 112 & & 912.766 & 900.136 \\
\hline 96 & 828.995 & 828.058 & 819.421 & 113 & & 913.513 & \\
\hline 97 & 834.571 & 832.570 & & 114 & & 919.775 & 907.646 \\
\hline 98 & 840.986 & 841.163 & 830.903 & 115 & & 920.192 & \\
\hline 99 & 845.394 & 845.227 & & 116 & & 925.786 & 914.714 \\
\hline 100 & 852.214 & 853.117 & 842.122 & 117 & & 925.900 & \\
\hline 101 & 857.073 & 857.372 & & 118 & & 931.356 & 921.459 \\
\hline 102 & 863.571 & 864.960 & 853.053 & 119 & & 930.747 & \\
\hline 103 & 867.871 & 868.673 & & 120 & & 935.292 & 927.963 \\
\hline 104 & 873.851 & 875.534 & 863.613 & 121 & & 934.062 & \\
\hline 105 & 877.664 & 879.316 & & 122 & & 938.468 & 934.285 \\
\hline 106 & 883.934 & 885.949 & 873.647 & & & & \\
\hline
\end{tabular}




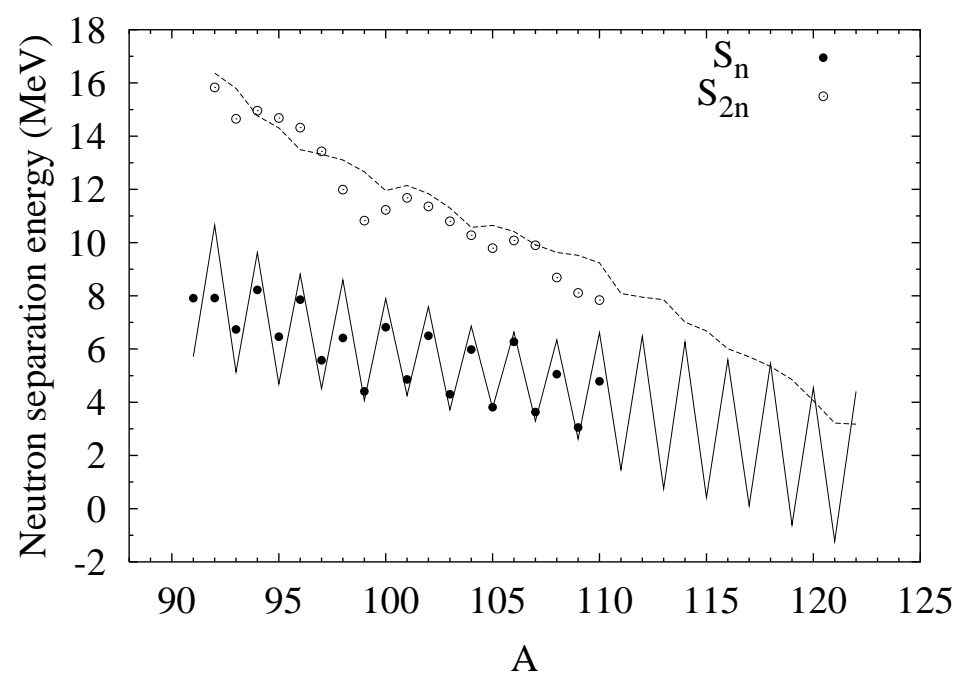

FIG. 1: Calculated and experimental one and two neutron separation energy in Zr isotopes. Theoretical results for one (two) neutron separation energies are connected by solid (dashed) lines while experimental values are indicated by hollow (filled) circles.

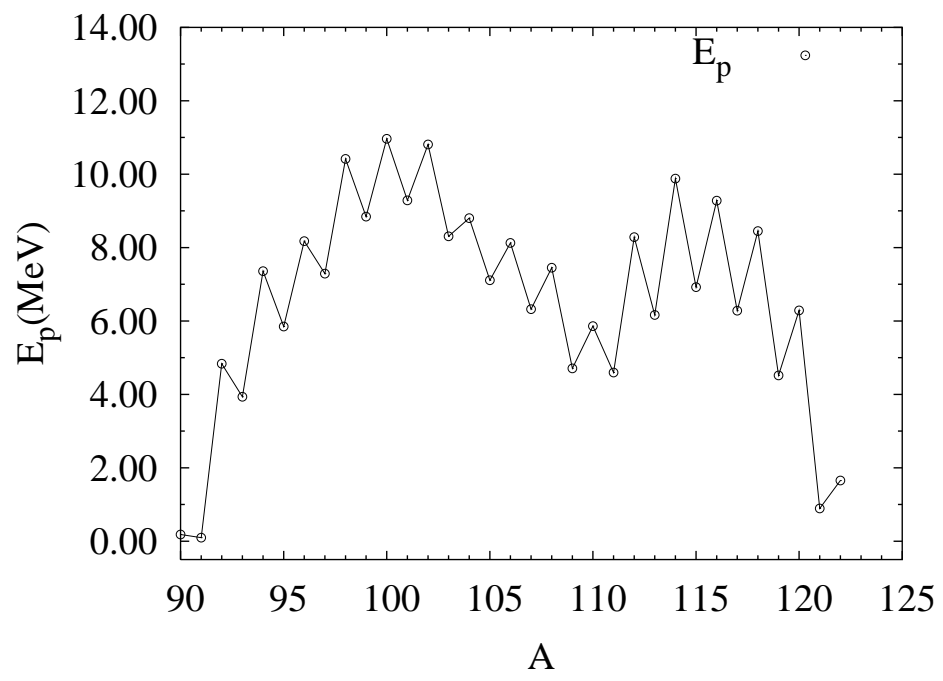




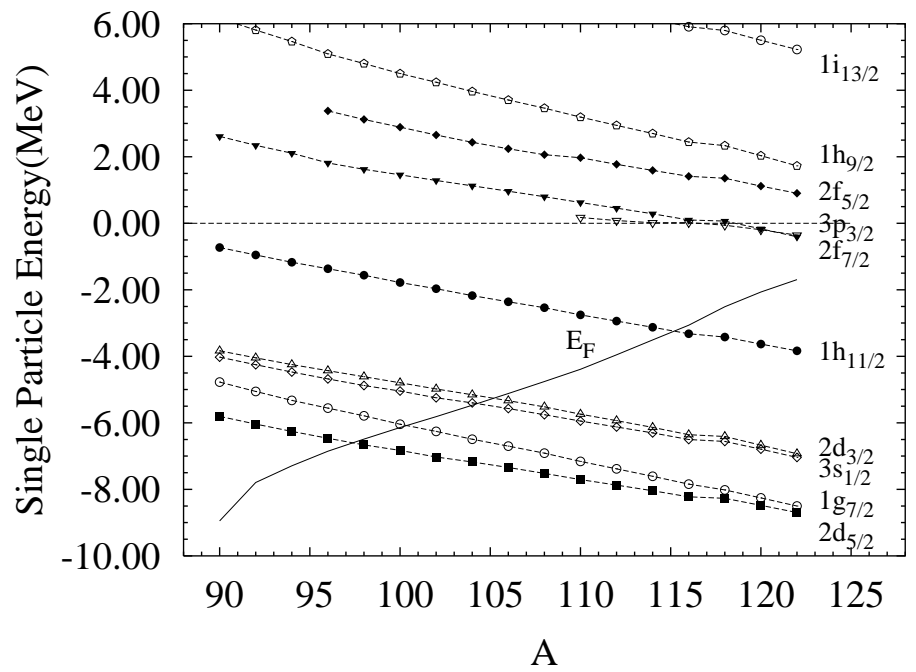

FIG. 3: Single particle levels in even $\mathrm{Zr}$ isotopes

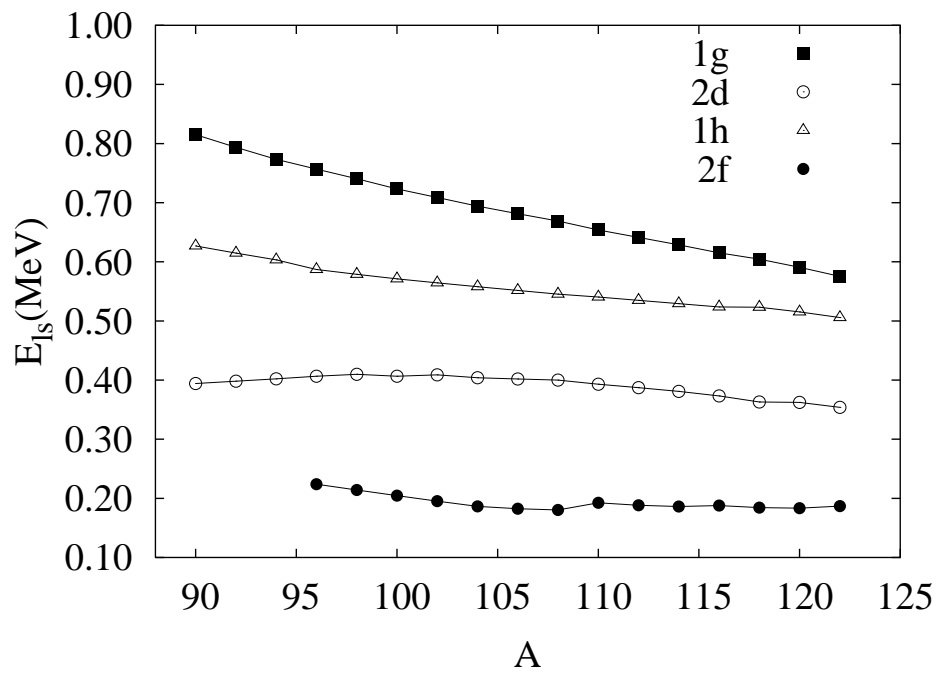

FIG. 4: Spin orbit splitting in even $\mathrm{Zr}$ isotopes 